\title{
Bianchi Type-II, VIII \& IX Perfect Fluid Cosmological Models in Brans Dicke Theory of Gravitation
}

\author{
Velagapudi Uma Maheswara Rao, Mandangi Vijaya Santhi \\ Department of Applied Mathematics, Andhra University, Visakhapatnam, India \\ E-mail:umrao57@hotmail.com \\ Received March 26, 2011; revised May 23, 2011; accepted June 6, 2011
}

\begin{abstract}
Field equations in the presence of perfect fluid distribution are obtained in a scalar tensor theory of gravitation proposed by Brans and Dicke [1] with the aid of Bianchi type-II, VIII \& IX metrics. Exact prefect fluid Bianchi type- IX cosmological model is presented since other models doesn't exist in Brans-Dicke scalar tensor theory of gravitation. Some physical and geometrical properties of the models are also discussed.
\end{abstract}

Keywords: Bianchi Type-II, VIII \& IX, Brans Dicke Scalar Tensor Theory, Perfect Fluid Distribution

\section{Introduction}

Brans and Dicke [1] theory of gravitation is well known modified version of Einstein's theory. It is a scalar tensor theory in which the gravitational interaction is mediated by a scalar field $\phi$ as well as the tensor field $g_{i j}$ of Einstein's theory. In this theory the scalar field $\phi$ has the dimension of the inverse of the gravitational constant.In recent years, there has been a renewed interest of the gravitational constant. The latest inflationary models (Mathiazhagan and Johri [2]), possible "graceful exit" problem (Pimental [3]) and extended chaotic inflations (Linde [4]) are based on Brans and Dicke theory of gravitation.

Brans-Dicke field equations for combined scalar and tensor field are

$$
\begin{aligned}
G_{i j}= & -8 \pi \phi^{-1} T_{i j}-\omega \phi^{-2}\left(\phi_{, i} \phi_{, j}-\frac{1}{2} g_{i j} \phi_{, k} \phi^{, k}\right) \\
& -\phi^{-1}\left(\phi_{i ; j}-g_{i j} \phi_{; k}{ }^{k}\right)
\end{aligned}
$$

and

$$
\phi=\phi_{; k}{ }^{, k}=8 \pi(3+2 \omega)^{-1} T
$$

where $G_{i j}=R_{i j}-\frac{1}{2} g_{i j} R$ is an Einstein tensor,

$T_{i j}$ is the stress energy tensor of the matter, $\omega$ is the dimensionless coupling constant and comma and semicolon denote partial and covariant differentiation respecttively.

The equation of motion

$$
T_{; j}^{i j}=0
$$

is a consequence of the field Equations (1.1) and (1.2).

Several aspects of Brans-Dicke cosmology have been extensively investigated by many authors. The work of Singh and Rai [5] gives a detailed discussion of BransDicke cosmological models. In particular, spatially homogeneous Bianchi models in Brans-Dicke theory in the presence of perfect fluid with or with out radiation are quite important to discuss the early stages of evolution of the universe.

Nariai [6], Belinskii and Khalatnikov [7], Reddy and Rao [8], Banerjee and Santos [9], Singh et al. [10], Shriram [11], Shriram and Singh [12], Berman et al. [13], Reddy [14], Reddy et al. [15], Adhav et al. [16] and Rao et al. $[17,18]$ are some of the authors who have investigated several aspects of this theory.

Chakraborty [19], Raj Bali and Dave [20], Raj Bali and Yadav [21] studied Bianchi type IX string as well as viscous fluid models in general relativity. Reddy, Patrudu and Venkateswarlu [22] studied Bianchi typeII, VIII \& IX models in scale covariant theory of gravitation. Shanthi and Rao [23] studied Bianchi type-VIII \& IX models in Lyttleton-Bondi Universe. Also Rao and Sanyasi Raju [24] and Sanyasi Raju and Rao [25] have studied Bianchi type-VIII \& IX models in Zero mass scalar fields and self creation cosmology. Rahaman et al. [26] have investigated Bianchi type-IX string cosmological model in a scalar-tensor theory formulated by Sen [27] based on Lyra [28] manifold. Rao et al. [29-31] have studied Bianchi type-II, VIII \& IX 
string cosmological models, perfect fluid cosmological models in SaezBallester scalar-tensor theory of gravitation and string cosmological models in general relativity as well as self creation theory of gravitation respectively.

In this paper we discuss Bianchi type-II, VIII \& IX perfect fluid cosmological models in a scalar-tensor theory proposed by Brans and Dicke [1].

\section{Metric and Energy Momentum Tensor}

We consider a spatially homogeneous Bianchi type-II, VIII and IX metrics of the form

$$
d s^{2}=d t^{2}-R^{2}\left[d \theta^{2}+f^{2}(\theta) d \phi^{2}\right]-S^{2}[d \psi+h(\theta) d \phi]^{2}
$$

where $(\theta, \phi, \psi)$ are the Eulerian angles, $R$ and $S$ are functions of $t$ only. It represents

Bianchi type-II if $f(\theta)=1$ and $h(\theta)=\theta$

Bianchi type-VIII if $f(\theta)=\cosh \theta$ and

$h(\theta)=\sinh \theta$

Bianchi type-IX if $f(\theta)=\sin \theta$ and $h(\theta)=\cos \theta$

The energy momentum tensor for perfect fluid distribution is given by

$$
T_{i j}=(\rho+p) u_{i} u_{j}-p g_{i j}
$$

where $\rho$ is the density and $p$ is the pressure.

Also

$$
g_{i j} u^{i} u^{j}=1
$$

In the co moving coordinate system, we have from Equations (2.2) and (2.3)

$$
T_{1}^{1}=T_{2}^{2}=T_{3}^{3}=-p, T_{4}^{4}=\rho \text { and } T_{i}^{i}=0 \text { for } i \neq j
$$

The quantities $\rho$ and $p$ are functions of " $t$ " only.

\section{Bianchi Type-II, VIII \& IX Perfect Fluidcosmological Models in Brans-Dicke Theory of Gravitation}

The field Equations (1.1), (1.2) \& (1.3) for the metric (2.1) with the help of Equations (2.2), (2.3) and (2.4) can be written as

$$
\begin{aligned}
& \frac{\ddot{R}}{R}+\frac{\ddot{S}}{S}+\frac{\dot{R} \dot{S}}{R S}+\frac{S^{2}}{4 R^{4}}+\frac{\omega \dot{\phi}^{2}}{2 \phi^{2}}+\frac{\ddot{\phi}}{\phi}+\frac{\dot{R} \dot{\phi}}{R \phi}+\frac{\dot{S} \dot{\phi}}{S \phi}=\frac{-8 \pi p}{\phi} \\
& \frac{2 \ddot{R}}{R}+\frac{\dot{R}^{2}+\delta}{R^{2}}-\frac{3 S^{2}}{4 R^{4}}+\frac{\omega \dot{\phi}^{2}}{2 \phi^{2}}+\frac{\ddot{\phi}}{\phi}+\frac{2 \dot{R} \dot{\phi}}{R \phi}=\frac{-8 \pi p}{\phi} \\
& \frac{2 \dot{\mathrm{R}} \dot{\mathrm{S}}}{\mathrm{RS}}-\frac{\mathrm{S}^{2}}{4 \mathrm{R}^{4}}+\frac{\dot{R}^{2}+\delta}{R^{2}}-\frac{\omega \dot{\phi}^{2}}{2 \phi^{2}}+\frac{2 \dot{R} \dot{\phi}}{R \phi}+\frac{\dot{S} \dot{\phi}}{S \phi}=\frac{8 \pi \rho}{\phi}
\end{aligned}
$$

$$
\begin{gathered}
\left(\frac{\dot{S}}{S}-\frac{\dot{R}}{R}\right) \frac{h(\theta) \dot{\phi}}{\phi}=0 \\
\ddot{\phi}+\dot{\phi}\left(\frac{2 \dot{R}}{R}+\frac{\dot{S}}{S}\right)=\frac{8 \pi}{3+2 \omega}(\rho-3 p)
\end{gathered}
$$

and

$$
\dot{\rho}+\left(\frac{2 \dot{R}}{R}+\frac{\dot{S}}{S}\right)(\rho+p)=0
$$

where ". " denotes differentiation with respect to " $t$ ".

When $\delta=0,-1 \&+1$, the field Equations (3.1)-(3.6) correspond to the Bianchi type-II, VIII \& IX universes respectively.

Using the transformation $R=e^{\alpha}, S=e^{\beta}, d t=R^{2} S d T$, where $\alpha$ and $\beta$ are functions of " $T$ " only.

The field Equations (3.1) to (3.6) reduce to

$$
\begin{aligned}
& \alpha^{\prime \prime}+\beta^{\prime \prime}-\alpha^{\prime^{2}}-2 \alpha^{\prime} \beta^{\prime}+\frac{e^{4 \beta}}{4}+\frac{\omega \phi^{\prime 2}}{2 \phi^{2}}-\frac{\alpha^{\prime} \phi^{\prime}}{\phi}+\frac{\phi^{\prime \prime}}{\phi} \\
& =\frac{-8 \pi p}{\phi} e^{(4 \alpha+2 \beta)} \\
& 2 \alpha^{\prime \prime}-\alpha^{\prime^{2}}-2 \alpha^{\prime} \beta^{\prime}+\delta e^{(2 \alpha+2 \beta)}-\frac{3}{4} e^{4 \beta}+\frac{\omega \phi^{\prime 2}}{2 \phi^{2}}-\frac{\beta^{\prime} \phi^{\prime}}{\phi}+\frac{\phi^{\prime \prime}}{\phi} \\
& =\frac{-8 \pi p}{\phi} e^{(4 \alpha+2 \beta)}
\end{aligned}
$$

$$
\begin{aligned}
& 2 \alpha^{\prime} \beta^{\prime}+\alpha^{\prime^{2}}+\delta e^{(2 \alpha+2 \beta)}-\frac{1}{4} e^{4 \beta}-\frac{\omega \phi^{\prime 2}}{2 \phi^{2}}+\frac{\beta^{\prime} \phi^{\prime}}{\phi}+\frac{2 \alpha^{\prime} \phi^{\prime}}{\phi} \\
& =\frac{8 \pi \rho}{\phi} e^{(4 \alpha+2 \beta)}
\end{aligned}
$$

$$
\begin{gathered}
\left(\alpha^{\prime}-\beta^{\prime}\right) \frac{h(\theta) \phi^{\prime}}{\phi}=0 \\
\phi^{\prime \prime}=\frac{8 \pi}{3+2 \omega}(\rho-3 p) e^{(4 \alpha+2 \beta)} \\
\rho^{\prime}+\left(2 \alpha^{\prime}+\beta^{\prime}\right)(\rho+p)=0
\end{gathered}
$$

where " " " denotes differentiation with respect to " $T$ ".

Since we are considering the Bianchi type-II, VIII and IX metrics, we have $h(\theta)=\theta, h(\theta)=\sinh \theta$ \&

$h(\theta)=\cos \theta$ for Bianchi type-II, VIII and IX metrics respectively. Therefore, from the Equation (3.10), we will consider the following possible cases with $h(\theta) \neq 0$.

1) $\alpha^{\prime}-\beta^{\prime}=0$ and $\phi^{\prime} \neq 0$

2) $\alpha^{\prime}-\beta^{\prime} \neq 0$ and $\phi^{\prime}=0$

3) $\alpha^{\prime}-\beta^{\prime}=0$ and $\phi^{\prime}=0$ 
CASE (1): $\alpha^{\prime}-\beta^{\prime}=0$ and $\phi^{\prime} \neq 0$ :

Here, we get $\alpha=\beta+c$

Without loss of generality by taking the constant of integration $c=0$, we get

$$
\alpha=\beta
$$

By using (3.13), the field Equations (3.7) to (3.12) will reduce to

$$
\begin{gathered}
2 \beta^{\prime \prime}-3 \beta^{\prime 2}+\frac{e^{4 \beta}}{4}+\frac{\omega \phi^{\prime 2}}{2 \phi^{2}}-\frac{\beta^{\prime} \phi^{\prime}}{\phi}+\frac{\phi^{\prime \prime}}{\phi}=\frac{-8 \pi p}{\phi} e^{6 \beta} \\
2 \beta^{\prime \prime}-3 \beta^{\prime 2}+\delta e^{4 \beta}-\frac{3}{4} e^{4 \beta}+\frac{\omega \phi^{\prime 2}}{2 \phi^{2}}-\frac{\beta^{\prime} \phi^{\prime}}{\phi}+\frac{\phi^{\prime \prime}}{\phi}=\frac{-8 \pi p}{\phi} e^{6 \beta} \\
3 \beta^{\prime 2}+\delta e^{4 \beta}-\frac{1}{4} e^{4 \beta}-\frac{\omega \phi^{\prime 2}}{2 \phi^{2}}+\frac{3 \beta^{\prime} \phi^{\prime}}{\phi}=\frac{8 \pi \rho}{\phi} e^{6 \beta} \\
\phi^{\prime \prime}=\frac{8 \pi}{3+2 \omega}(\rho-3 p) e^{6 \beta} \\
\rho^{\prime}+(\rho+p) 3 \beta^{\prime}=0
\end{gathered}
$$

where " ' " denotes differentiation with respect to " $T$ ".

From (3.14) and (3.15), we have

$$
\delta e^{4 \beta}-e^{4 \beta}=0
$$

From (3.19), we observe that, we can't find Bianchi type-II ( $\delta=0)$ and VIII $(\delta=-1)$ perfect fluid cosmological models in Brans-Dicke theory of gravitation. But we can get Bianchi type-IX $(\delta=1)$ perfect fluid cosmological model in Brans-Dicke theory of gravitation.

For $\delta=1$, the field Equations (3.14)-(3.18) reduce to

$$
\begin{gathered}
2 \beta^{\prime \prime}-3 \beta^{\prime 2}+\frac{e^{4 \beta}}{4}++\frac{\omega \phi^{\prime 2}}{2 \phi^{2}}-\frac{\beta^{\prime} \phi^{\prime}}{\phi}+\frac{\phi^{\prime \prime}}{\phi}=\frac{-8 \pi p}{\phi} e^{4 \beta} \\
3 \beta^{\prime 2}+\frac{3}{4} e^{4 \beta}-\frac{\omega \phi^{\prime 2}}{2 \phi^{2}}+\frac{3 \beta^{\prime} \phi^{\prime}}{\phi}=\frac{8 \pi \rho}{\phi} e^{4 \beta} \\
\phi^{\prime \prime}=\frac{8 \pi}{3+2 \omega}(\rho-3 p) e^{6 \beta} \\
\rho^{\prime}+3(\rho+p) \beta^{\prime}=0
\end{gathered}
$$

From (3.20), (3.21) \& (3.23), we get

$$
\beta^{\prime \prime}-\beta^{\prime 2}+\frac{e^{4 \beta}}{4}+\frac{\omega \phi^{\prime 2}}{6 \phi^{2}}-\frac{\omega \phi^{\prime \prime}}{3 \phi}=0
$$

Then from (3.24), we get

$$
\begin{gathered}
\phi=(a T+b)^{-2} \\
e^{\beta}=(a T+b)^{-1 / 2}=e^{\alpha}
\end{gathered}
$$

with the relation $16 a^{2} \omega-3 a^{2}-3=0$, where $a \& b$ are arbitrary constants.

Using (3.25) \& (3.26) in (3.20) \& (3.21), we get

$$
\begin{aligned}
& 8 \pi p=\frac{\left(11 a^{2}-8 a^{2} \omega-1\right)(a T+b)^{-1}}{4} \\
& 8 \pi \rho=\frac{\left(15 a^{2}+3-8 a^{2} \omega\right)(a T+b)^{-1}}{4}
\end{aligned}
$$

The corresponding metric can be written in the form

$$
\begin{aligned}
d s^{2}= & (a T+b)^{-3} d T^{2}-(a T+b)^{-1}\left[d \theta^{2}+\sin ^{2} \theta d \varphi^{2}\right] \\
& -(a T+b)^{-1}[d \psi+\cos \theta d \varphi]^{2}
\end{aligned}
$$

Thus (3.29) together with (3.27) and (3.28) constitutes an exact Bianchi type-IX perfect fluid cosmological model in Brans-Dicke scalar-tensor theory of gravitation.

PHYSICAL AND GEOMETRICAL PROPERTIES:

The volume element of the Bianchi type-IX perfect fluid cosmological model is given by

$$
V=(-g)^{\frac{1}{2}}=(a T+b)^{\frac{-3}{2}} \sin \theta
$$

We can observe that the spatial volume $V$ decreases as time " $T$ " increases, i.e., the model is contracting. Also the model has initial singularity at $T=-b / a, a \neq 0$

The scalar expansion $\theta$ and shear $\sigma$ are given by

$$
\begin{gathered}
\theta=u_{; i}{ }^{i}=\frac{-3 a}{2(a T+b)} \\
\sigma^{2}=\frac{9 a^{2}}{24(a T+b)^{2}}
\end{gathered}
$$

for Bianchi type-IX perfect fluid cosmological model in Brans-Dicke theory of gravitation. The scalar expansion $\theta \rightarrow 0$ as $T \rightarrow \infty$ and $\theta \rightarrow \infty$ as $T \rightarrow 0$. So, the rate of expansion is rapid as time decreases and it becomes slow as time increases. The shear scalar $\sigma^{2} \rightarrow 0$ as $T \rightarrow \infty$ and $\sigma^{2} \rightarrow \infty$ as $T \rightarrow 0$. Thus the shape of universe changes uniformly. The deceleration parameter $q$ is obtained as $q=-3$. The negative value of $q$ indicates that the model is inflationary. Since $\lim _{T \rightarrow \infty}\left(\frac{\sigma}{\theta}\right) \neq 0$ which confirms that the universe remains anisotropic throughout the evolution.

CASE (2): $\alpha^{\prime}-\beta^{\prime} \neq 0$ and $\phi^{\prime}=0$ :

In this case $\phi=c_{1}$, where $c_{1}$ is a constant of integration, without loss of generality we can take $c_{1}=1$.

Hence the field Equations (3.7) to (3.12) reduce to general relativity field equations with $\alpha \neq \beta$.

$$
\alpha^{\prime \prime}+\beta^{\prime \prime}-\alpha^{\prime^{2}}-2 \alpha^{\prime} \beta^{\prime}+\frac{e^{4 \beta}}{4}=-8 \pi p e^{(4 \alpha+2 \beta)}
$$




$$
\begin{gathered}
2 \alpha^{\prime \prime}-\alpha^{\prime^{2}}-2 \alpha^{\prime} \beta^{\prime}+\delta e^{(2 \alpha+2 \beta)}-\frac{3}{4} e^{4 \beta}=-8 \pi p e^{(4 \alpha+2 \beta)} \\
2 \alpha^{\prime} \beta^{\prime}+\alpha^{\prime^{2}}+\delta e^{(2 \alpha+2 \beta)}-\frac{1}{4} e^{4 \beta}=8 \pi \rho e^{(4 \alpha+2 \beta)} \\
0=\frac{8 \pi}{3+2 \omega}(\rho-3 p) e^{(4 \alpha+2 \beta)} \\
\rho^{\prime}+\left(2 \alpha^{\prime}+\beta^{\prime}\right)(\rho+p)=0
\end{gathered}
$$

From (3.33), we get

$$
\rho=3 p
$$

Since $\rho=3 p$, we will get only radiating universe in this case.

The field Equations (3.30) to (3.34) reduce to

$$
\begin{gathered}
\alpha^{\prime \prime}+\beta^{\prime \prime}-\alpha^{\prime^{2}}-2 \alpha^{\prime} \beta^{\prime}+\frac{e^{4 \beta}}{4}=-8 \pi p e^{(4 \alpha+2 \beta)} \\
2 \alpha^{\prime \prime}-\alpha^{\prime^{2}}-2 \alpha^{\prime} \beta^{\prime}+\delta e^{(2 \alpha+2 \beta)}-\frac{3}{4} e^{4 \beta}=-8 \pi p e^{(4 \alpha+2 \beta)} \\
2 \alpha^{\prime} \beta^{\prime}+\alpha^{\prime^{2}}+\delta e^{(2 \alpha+2 \beta)}-\frac{1}{4} e^{4 \beta}=24 \pi p e^{(4 \alpha+2 \beta)} \\
\rho^{\prime}+\frac{4}{3} \rho\left(2 \alpha^{\prime}+\beta^{\prime}\right)=0
\end{gathered}
$$

From (3.36) to (3.38), we have

$$
\alpha^{\prime \prime}+2 \beta^{\prime \prime}-\alpha^{\prime^{2}}-2 \alpha^{\prime} \beta^{\prime}+\frac{3 e^{4 \beta}}{4}=0
$$

Then from (3.40), we get

$$
\begin{aligned}
e^{\alpha} & =(a T+b)^{m} \\
e^{\beta} & =(a T+b)^{1 / 2}
\end{aligned}
$$

where $m, a$ and $b$ are arbitrary constants satisfying $4 a^{2}\left(m^{2}-1\right)=3, a \neq 0 \& m^{2} \neq 1$.

FOR BIANCHI TYPE- II METRIC $(\delta=0)$ :

From (3.36)-(3.38), we get

$$
\begin{aligned}
& 8 \pi p=\frac{\left(2 m^{2} a^{2}+4 m a^{2}+1\right)}{4(a T+b)^{4 m+1}} \\
& 8 \pi \rho=\frac{\left(4 m^{2} a^{2}-4 m a^{2}-1\right)}{4(a T+b)^{4 m+1}}
\end{aligned}
$$

The corresponding metric can be written in the form

$$
\begin{aligned}
d s^{2}= & (a T+b)^{4 m-1} d T^{2}-(a T+b)^{2 m}\left[d \theta^{2}+d \varphi^{2}\right] \\
& -(a T+b)^{-1}[d \psi+\theta d \varphi]^{2}
\end{aligned}
$$

Thus (3.45) together with (3.43) \& (3.44) constitutes Bianchi type-II Perfect fluid radiating cosmological models in general theory of relativity.

FOR BIANCHI TYPE-VIII METRIC $(\delta=-1)$ :

From (3.36)-(3.38), we get

$$
\begin{aligned}
& 8 \pi p=\frac{\left(4 m^{2} a^{2}+4 m a^{2}+4(a T+b)^{2 m+1}+3\right)}{4(a T+b)^{4 m+1}} \\
& 8 \pi \rho=\frac{\left(4 m^{2} a^{2}-4 m a^{2}-4(a T+b)^{2 m+1}-1\right)}{4(a T+b)^{4 m+1}}
\end{aligned}
$$

The corresponding metric can be written in the form

$$
\begin{aligned}
d s^{2}= & (a T+b)^{4 m-1} d T^{2} \\
& -(a T+b)^{2 m}\left[d \theta^{2}+\cosh ^{2} \theta d \phi^{2}\right] \\
& -(a T+b)^{-1}[d \psi+\sinh \theta d \phi]^{2}
\end{aligned}
$$

Thus (3.48) together with (3.46) \& (3.47) constitutes Bianchi type-VIII Perfect fluid radiating cosmological models in general theory of relativity.

FOR BIANCHI TYPE-IX METRIC $(\delta=1)$ :

From (3.36)-(3.38), we get

$$
\begin{aligned}
& 8 \pi p=\frac{\left(4 m^{2} a^{2}+4 m a^{2}-4(a T+b)^{2 m+1}+3\right)}{4(a T+b)^{4 m+1}} \\
& 8 \pi \rho=\frac{\left(4 m^{2} a^{2}-4 m a^{2}+4(a T+b)^{2 m+1}-1\right)}{4(a T+b)^{4 m+1}}
\end{aligned}
$$

The corresponding metric can be written in the form

$$
\begin{aligned}
d s^{2}= & (a T+b)^{4 m-1} d T^{2} \\
& -(a T+b)^{2 m}\left[d \theta^{2}+\sin ^{2} \theta d \phi^{2}\right] \\
& -(a T+b)^{-1}[d \psi+\cos \theta d \phi]^{2}
\end{aligned}
$$

Thus (3.51) together with (3.49) \& (3.50) constitutes Bianchi type-IX Perfect fluid radiating cosmological models in general theory of relativity.

PHYSICAL AND GEOMETRICAL PROPERTIES:

The volume element of the above three models [(3.45), (3.48) \& (3.51)] are given by

$$
V=(-g)^{\frac{1}{2}}=(a T+b)^{\frac{4 m-1}{2}} f(\theta)
$$

where $f(\theta)=1, \sinh \theta$ and $\sin \theta$ respectively.

In the above expressions, the volume decreases as time increases if $m<1 / 4$ i.e., the models are contracting, the volume increases as time increases if $m>1 / 4$ i.e., the models are expanding and the volume is independent of time $T$ if $m=1 / 4$. Also the models have initial singular-- ity at $T=-b / a, a \neq 0$. 
The expansion $\theta$ and shear $\sigma$ are equal for all $\mathrm{Bi}-$ anchi type-II, VIII \& IX perfect fluid radiating cosmological models in general relativity. Which are given by

$$
\begin{gathered}
\theta=u_{; i}^{i}=\frac{a(4 m-1)}{2(a T+b)} \\
\sigma^{2}=\frac{a^{2}(4 m-1)^{2}}{12(a T+b)^{2}}
\end{gathered}
$$

The deceleration parameter

$$
q=-3 \theta^{-2}\left(\theta_{; i} u^{i}+\frac{1}{3} \theta^{2}\right)=\left(\frac{7-4 m}{4 m-1}\right)
$$

It can be seen that for large " $T$ " the quantities $\theta$ and $\sigma$ will become zero if $m \neq 1 / 4$. Also the quantities $\theta$ and $\sigma$ tends to $+\infty$ as $T \rightarrow 0$ if $4 m-1>0$ and tends to $-\infty$ if $4 m-1<0$. Thus the rate of expansion is rapid as time decreases, it becomes slow as time increases and the shape of universe changes uniformly. In the case of $4 m-1=0$, we can see that the Spatial Volume " $V$ " is independent of time " $T$ " and $\theta, \sigma$ will become zero. Also, since $\lim _{T \rightarrow \infty}\left(\frac{\sigma}{\theta}\right) \neq 0$, the models are not isotropic for large $T$. The negative value of the deceleration parameter $q$ shows that the models inflate except for $m=1$.

CASE (3): $\alpha^{\prime}-\beta^{\prime}=0$ and $\phi^{\prime}=0$

Here, we get $\alpha=\beta+c$

Without loss of generality by taking the constant of integration $c=0$, we get

$$
\alpha=\beta
$$

Since $\phi^{\prime}=0 \Rightarrow \phi=c$,

where $c$ is a constant of integration, without loss of generality we can take $c=1$.

Hence the field equations (3.7) to (3.12) reduce to general relativity field equations with $\alpha=\beta$.

$$
\begin{gathered}
2 \beta^{\prime \prime}-3 \beta^{\prime 2}+\frac{e^{4 \beta}}{4}=-8 \pi p e^{6 \beta} \\
2 \beta^{\prime \prime}-3 \beta^{\prime 2}+\delta e^{4 \beta}-\frac{3}{4} e^{4 \beta}=-8 \pi p e^{6 \beta} \\
3 \beta^{\prime 2}+\delta e^{4 \beta}-\frac{1}{4} e^{4 \beta}=8 \pi \rho e^{6 \beta} \\
0=\frac{8 \pi}{3+2 \omega}(\rho-3 p) e^{6 \beta} \\
\rho^{\prime}+(\rho+p) 3 \beta^{\prime}=0
\end{gathered}
$$

where " ' " denotes differentiation with respect to " $T$ ".

From (3.53) and (3.54), we have

$$
\delta e^{4 \beta}-e^{4 \beta}=0
$$

From (3.58), we observe that, we can't find Bianchi type II $(\delta=0)$ and VIII $(\delta=-1)$ perfect fluid cosmological models of general relativity. But we can get only Bianchi type IX $(\delta=1)$ perfect fluid cosmological model of general relativity.

For $\delta=1$, the field equations (3.53)-(3.57) reduce to

$$
\begin{gathered}
2 \beta^{\prime \prime}-3 \beta^{\prime 2}+\frac{e^{4 \beta}}{4}=-8 \pi p e^{4 \beta} \\
3 \beta^{\prime 2}+\frac{3}{4} e^{4 \beta}=8 \pi \rho e^{4 \beta} \\
0=\frac{8 \pi}{3+2 \omega}(\rho-3 p) e^{6 \beta} \\
\rho^{\prime}+(\rho+p) 3 \beta^{\prime}=0
\end{gathered}
$$

From (3.61), we get

$$
\rho=3 p
$$

Since from $\rho=3 p$, we will get only radiating universe in this case.

Now from (3.59), (3.60) and (3.61), we have

$$
\beta^{\prime \prime}-\beta^{2}+\frac{e^{4 \beta}}{4}=0
$$

From (3.64), we get

$$
e^{2 \beta}=4\left[(a T+b)^{2}+4\right]^{-1}
$$

Using (3.65) in (3.59) \& (3.60), we get

$$
8 \pi \rho=24 \pi p=\frac{3(a T+b)^{4}+24(a T+b)^{2}+48}{64}
$$

The corresponding metric can be written in the form

$$
\begin{aligned}
d s^{2}= & 64\left[(a T+b)^{2}+4\right]^{-3} d T^{2} \\
& -4\left[(a T+b)^{2}+4\right]^{-1}\left[d \theta^{2}+\sin ^{2} \theta d \phi^{2}\right] \\
& -4\left[(a T+b)^{2}+4\right]^{-1}[d \psi+\cos \theta d \phi]^{2}
\end{aligned}
$$

Thus (3.67) together with (3.66) constitutes Bianchi type-IX radiating perfect fluid cosmological model in general theory of relativity.

PHYSICAL AND GEOMETRICAL PROPERTIES:

The volume element of the model (3.67) is given by

$$
V=(-g)^{\frac{1}{2}}=64\left[(a T+b)^{2}+4\right]^{-3} \sin \theta
$$

Now the expression for expansion $\theta$ and shear $\sigma$ are given by

$$
\theta=u_{; i}^{i}=\frac{-6 a(a T+b)}{\left((a T+b)^{2}+4\right)}
$$




$$
\sigma^{2}=\frac{6 a^{2}(a T+b)^{2}}{\left((a T+b)^{2}+4\right)^{2}}
$$

for Bianchi type-IX perfect fluid radiating cosmological model in Brans-Dicke theory of gravitation. The spatial volume tends to zero as $T \rightarrow \infty$. Thus the model is contracting with the increase of time and also the model has no real singularity. The deceleration parameter $\mathrm{q}$ is obtained as $q=-2$. The negative value of $q$ indicates that the model is inflationary. Also, since $\lim _{T \rightarrow \infty}\left(\frac{\sigma}{\theta}\right) \neq 0$

which confirms that the universe remains anisotropic throughout the evolution.

\section{Conclusions}

Bianchi type space-times play a vital role in understanding and description of the early stages of evolution of the universe. In particular, the study of Bianchi type-II, VIII \& IX universes are important because familiar solutions like FRW universe with positive curvature, the desitter universe, the Taub-Nut solutions etc correspond of Bianchi type-II, VIII \& IX space-times. In view of the importance of Bianchi type-II, VIII \& IX space- times and also since exact solutions offer an alternative and complementary approach to study various cosmological models, in this paper we have presented Bianchi type-II, VIII \& IX perfect fluid cosmological models in BransDicke theory of gravitation.

In case of $\alpha^{\prime}-\beta^{\prime}=0$ and $\phi^{\prime} \neq 0$, we can observe that the only Bianchi type-IX perfect fluid cosmological model exists in Brans-Dicke theory of gravitation. The model is anisotropic, inflationary and has initial singularity at $T=-b / a, a \neq 0$. Also established the non-existence of Bianchi type-II \& VIII perfect fluid cosmological models in this theory. Since " $a$ " is an arbitrary constant and " $\omega$ " is a coupling constant, it is always possible to assign specific values to " $a$ " and " $\omega$ " to keep the pressure " $p$ " (3.27) and density " $\rho$ " (3.28) be always positive.

In case of $\alpha^{\prime}-\beta^{\prime} \neq 0$ and $\phi^{\prime}=0$, we can observe that Bianchi type-II, VIII \& IX perfect fluid radiating cosmological models of general relativity exist in this theory. The models have initial singularity at $T=-b / a$, $a \neq 0$ and remain anisotropic throughout the evolution.

In case of $\alpha^{\prime}-\beta^{\prime}=0$ and $\phi^{\prime}=0$, we have obtained only Bianchi type-IX anisotropic radiating perfect fluid cosmological model of general relativity with $\alpha=\beta$. In this case also we have observed that Bianchi type-II \& VIII cosmological models doesn't exist in this theory.

\section{References}

[1] H. Brans and R. H. Dicke, "Mach's Principle and a Rela- tivistic Theory of Gravitation," Physical Review A, Vol. 124, No. 3, 1961, pp.925-935.

doi:10.1103/PhysRev.124.925

[2] C. Mathiazhagan and V. B. Johri, "An Inflationary Universe in Brans-Dicke Theory: A Hopeful Sign of Theoretical Estimation of the Gravitational Constant," Classical and Quantum Gravity, Vol. 1, No.2, 1984, pp. L29L32. doi:10.1088/0264-9381/1/2/005

[3] L. O. Pimentel, "New Exact Vacuum Solutions in BransDicke Theory," Modern Physics Letters A, Vol. 12, No. 25, 1997, pp. 1865-1870. doi:10.1142/S0217732397001904

[4] A. D. Linde, "Extended Chaotic Inflation and Spatial Variations of the Gravitational Constant," Physics Letters $B$, Vol. 238, No. 2-4, 1990, pp. 160-165. doi:10.1016/0370-2693(90)91713-L

[5] T. Singh and L. N. Rai, "Scalar-Tensor Theories of Gravitation: Foundations and Prospects," General Relativity and Gravitation, Vol. 15, No. 9, 1983, pp. 875-902. doi:10.1007/BF00778798

[6] H. Nariai, "Hamiltonian Approach to the Dynamics of Expanding Homogeneous Universes in the Brans-Dicke Cosmology," Progress of Theoretical Physics, Vol. 47, No. 6, 1972, pp. 1824-1843. doi:10.1143/PTP.47.1824

[7] V. A. Belinskii and I. M. Khalatnikov, "Effect of Scalar and Vector Fields on the Nature of the Cosmological Singularity," Soviet Physics-JETP, Vol. 36, No. 4, 1973, pp. 591-597.

[8] D. R. K. Reddy and V. U. M. Rao, "Field of a Charged Particle in Brans-Dicke Theory of Gravitation," Journal of Physics A: Mathematical and General, Vol. 14, No. 8, 1981, pp. 1973-1976. doi:10.1088/0305-4470/14/8/021

[9] A. Banerjee and N.O. Santos, "Bianchi Type-II Cosmological Models in Brans-Dicke Theory," Il Nuovo Cimento B, Vol. 67, No. 1, 1982, pp. 31-40. doi:10.1007/BF02721068

[10] T. Singh, L. N. Rai and T. Singh, "An Anisotropic Cosmological Model in Brans-Dicke Theory," Astrophysics and Space Science, Vol. 96, No. 1, 1983, pp. 95-105.

[11] S. Ram, "Spatially Homogeneous and Anisotropic Cosmological Solution in Brans-Dicke Theory," General Relativity and Gravitation, Vol. 15, No. 7, 1983, pp. 635640. doi:10.1007/BF00759040

[12] S. Ram and D. K. Singh, "LRS Bianchi Type-V Vacuum Cosmological Solution in Brans-Dicke Theory," Astrophysics and Space Science, Vol. 98, No. 1, 1984, pp. 193196. doi:10.1007/BF00651959

[13] M. S. Berman, M. M. Som and F. M. Gomide, "BransDicke Static Universes," General Relativity and Gravitation, Vol. 21, No. 3, 1989, pp. 287-292. doi:10.1007/BF00764101

[14] D. R. K. Reddy, "A String Cosmological Model in a Scalar-Tensor Theory of Gravitation," Astrophysics and Space Science, Vol. 286, No. 3-4, 2003, pp. 359-363. doi:10.1023/A:1026370732619 
[15] D. R. K. Reddy and R. L. Naidu, "Five Dimensional String Cosmological Models in a Scalar-Tensor Theory of Gravitation," Astrophysics and Space Science, Vol. 307, No. 4, 2007, pp. 395-398. doi:10.1007/s10509-007-9387-x

[16] K. S. Adhav, A. S. Nimkar and M. V. Dawande, "NDimensional String Cosmological Model in Brans-Dicke Theory of Gravitation," Astrophysics and Space Science, Vol. 310, No. 3-4, 2007, pp. 231-235. doi:10.1007/s10509-007-9506-8

[17] V. U. M. Rao, T. Vinutha, M. V. Shanthi and K. V. S. Sireesha, "Exact Bianchi Type-V Perfect Fluid Cosmological Models in Brans-Dicke Theory of Gravitation," Astrophysics and Space Science, Vol. 315, No. 1-4, 2008, pp. 211-214. doi:10.1007/s10509-008-9820-9

[18] V. U. M. Rao, T. Vinutha and M. V. Santhi, "Bianchi Type-V Cosmological Model with Perfect Fluid Using Negative Constant Deceleration Parameter in a Scalar Tensor Theory Based on Lyra Manifold," Astrophysics and Space Science, Vol. 314, No. 1-3, 2008, pp. 213-216. doi:10.1007/s10509-008-9757-z

[19] S. Chakraborty, "A Study on Bianchi-IX Cosmological Model," Astrophysics and Space Science, Vol. 180, No. 2, 1991, pp. 293-303. doi:10.1007/BF00648184

[20] R. Bali and S. Dave, "Bianchi Type IX String Cosmological Model in General Relativity," Pramana Journal of Physics, Vol. 56, No. 4, 2001, pp. 513-518. doi:10.1007/s12043-001-0100-2

[21] R. Bali and M. K. Yadav, "Bianchi Type-IX Viscous Fluid Cosmological Model in General Relativity," Pramana Journal of Physics, Vol. 64, No. 2, 2005, pp. 187196. doi:10.1007/BF02704873

[22] D. R. K. Reddy, B. M. Patrudu and R. Venkateswarlu, "Exact Bianchi Type-II, VIII and IX Cosmological Models in Scale-Covariant Theory of Gravitation," Astrophysics and Space Science, Vol. 204, No. 1, 1993, pp. 155-160. doi:10.1007/BF00658101

[23] K. Shanthi and V. U. M. Rao, "Bianchi Type-II and III
Models in Self-Creation Cosmology," Astrophysics and Space Science, Vol. 179, No.1, 1991, pp. 147-153. doi:10.1007/BF00642359

[24] V. U. M. Rao and Y. V. S. S. Sanyasiraju, "Exact Bianchi-Type VIII and IX Models in the Presence of ZeroMass Scalar Fields," Astrophysics and Space Science, Vol. 187, No. 1, 1992, pp.113-117. doi:10.1007/BF00642691

[25] Y. V. S. S. Sanyasiraju and V. U. M. Rao, "Exact Bianchi-Type VIII and IX Models in the Presence of the Self-Creation Theory of Cosmology," Astrophysics and Space Science, Vol. 189, No. 1, 1992, pp. 39-43. doi:10.1007/BF00642950

[26] F. Rahaman, S. Chakraborty, N. Begum, M. Hossain and M. Kalam, "Bianchi-IX String Cosmological Model in Lyra Geometry," Pramana Journal of Physics, Vol. 60, No. 6, 2003, pp. 1153-1159. doi:10.1007/BF02704282

[27] D. K. Sen, “A Static Cosmological Model," Zeitschrift for Physics A, Vol. 149, No. 3, 1957, pp. 311-323.

[28] G. Lyra, "Über eine Modifikation der Riemannschen Geometrie," Mathematische Zeitschrift, Vol. 54, No. 1, 1951, pp. 52-64. doi:10.1007/BF01175135

[29] V. U. M. Rao, M. V. Santhi and T. Vinutha, "Exact Bianchi Type-II, VIII and IX String Cosmological Models in Saez-Ballester Theory of Gravitation," Astrophysics and Space Science, Vol. 314, No. 1-3, 2008, pp. 73-77. doi:10.1007/s10509-008-9739-1

[30] V. U. M. Rao, M. V. Santhi and T. Vinutha, "Exact Bianchi Type-II, VIII and IX Perfect Fluid Cosmological Models in Saez-Ballester Theory of Gravitation," Astrophysics and Space Science, Vol. 317, No. 1-2, 2008, pp. 27-30. doi:10.1007/s10509-008-9849-9

[31] V. U. M. Rao, M. V. Santhi and T. Vinutha, "Exact Bianchi Type-II, VIII and IX String Cosmological Models in General Relativity and Self-Creation Theory of Gravitation," Astrophysics and Space Science, Vol. 317, No. 1-2, 2008, pp. 83-88. doi:10.1007/s10509-008-9859-7 